\title{
Más allá del capital social. La producción de hábitat popular en Buenos Aires*
}

\author{
Mariano Scheinsohn \\ Universidad de Buenos Aires, Buenos Aires, Argentina. Email: \\ marianoscheinsohn@yahoo.com.ar \\ Cecilia Cabrera
}

Universidad de Buenos Aires, Buenos Aires, Argentina. Email: ceciliac1@yahoo.com

Resumen: El concepto de capital social se encuentra ampliamente difundido en Latinoamérica -a escala local, nacional y supra-nacional- como fundamento y recomendación en la aplicación de políticas sociales destinadas a la disminución de la pobreza, entre las que se encuentran los programas y emprendimientos de vivienda social. La intención del presente artículo es señalar los límites que la disparidad de definiciones y el uso ambiguo de este término impone a su capacidad interpretativa y explicativa en relación con los complejos procesos sociales en que se inscribe. Se intentará ejemplificar esta cuestión a partir de la interpretación y análisis de dos experiencias relevantes en la producción de hábitat popular que se encuentran en curso en la ciudad de Buenos Aires. De las mismas se desprenden que la capacidad de movilización y disponibilidad del recurso vinculado al capital social implica necesariamente una apuesta de luchas en donde lo político juega un rol relevante. popular.

Palabras clave: Capital Social, Capital Político, Movimiento social, Hábitat

\section{Beyond social capital. The production of popular housing in Buenos Aires}

\begin{abstract}
In Latin America, "social capital” is a concept which is widely used -at local, national and supra-national level- as a fundamental recommendation to support social policies on poverty reduction and social housing. The intention of this paper is to point out the limits that the disparity in definitions and the ambiguous use of the term imposes on its interpretative and explanatory ability, in relation to the very complex social processes in which it is enrolled. It tries to exemplify this point analyzing two relevant social housing projects that are ongoing in the city of Buenos Aires. From that interpretation, it concludes mobilization capacity and resource availability linked to social capital, involve political struggles and play an important role in the effectiveness of that process.
\end{abstract} housing.

Key words: Social Capital, Political Capital, Social movement, Social 


\title{
Além do capital social. A produção de habitação popular em Buenos Aires
}

\begin{abstract}
Resumo: O conceito de capital social é amplamente difundido na América Latina -em níveles local, nacional e supra-nacionais-, como a fundação e recomendação em a implementação de políticas sociais que visem a redução da pobreza, entre os quais estão os programas e empreendimentos habitacionais de carácter social. A intenção deste artigo é apontar os limites que a disparidade de definições e do uso ambíguo do termo determina em sua capacidade de atuar e explicar em relação aos processos sociais complexos em que se inscrever. Esta questão é ilustrada a partir da interpretação e análise de duas experiências relevantes na produção de habitação popular as quais estám em andamento na cidade de Buenos Aires. Das mesmas mostram que a capacidade de mobilização e disponibilidade de recursos ligados ao capital social envolve necessariamente um compromisso das lutas políticas que desempenha um papel relevante.
\end{abstract}

Palavras-chave: Capital Social, Capital político, o movimento social, Habitat popular.

\section{Introducción}

En la última década, las principales instituciones internacionales dedicadas a promover políticas sociales a escala regional y los ámbitos gubernamentales de los países en donde estas se aplican, han utilizado y aceptado ampliamente el concepto de capital social (con diversas definiciones) como una de las estrategias prevalentes en las políticas destinadas a la reducción de la pobreza.

Así mismo, en los últimos tiempos, en diversos ámbitos institucionales y académicos han sido ampliamente documentados los límites y obstáculos que dicha estrategia ha presentado, especialmente en Latinoamérica, para una eficaz disminución a gran escala de la pobreza y la desigualdad social ${ }^{1}$.

El presente artículo desarrollará como eje principal de su análisis, la hipótesis de que el "capital político" constituiría un elemento conceptual relevante a considerar tanto en el análisis como en los procesos de producción de hábitat popular y en la promoción del desarrollo urbano integral, a partir del análisis socio-urbano de los casos de producción de hábitat popular en la ciudad de Buenos Aires llevados a cabo recientemente por movimientos sociales y de derechos humanos -que en su conjunto constituyen una de las más importantes intervenciones en la producción de vivienda social en el ámbito de la ciudad-.

Desde esta perspectiva dicho concepto englobaría un conjunto, poco analizado hasta ahora, de recursos estratégicos que los movimientos sociales poseen para la generación de condiciones de eficacia en las intervenciones y acciones sociales “a escala” en los procesos de producción de hábitat 
popular y en la reducción de la pobreza y la desigualdad social en Latinoamérica.

En definitiva, el objetivo principal de este trabajo, es proponer nuevos desarrollos conceptuales que, generados a partir de la investigación y análisis de casos, permitan aportar una visión más integral respecto de las posibilidades del desarrollo urbano desde el accionar de los movimientos sociales.

\section{De qué no se habla cuando se habla de Capital Social}

En los últimos años, la noción de capital social ha sido muy difundida tanto en el ámbito de la investigación como en la elaboración y prescripción de políticas sociales.

En Latinoamérica especialmente, ha tenido un amplio predicamento en la elaboración, diseño y aplicación de políticas sociales, tanto en ámbitos gubernamentales como en ONG's y diversas organizaciones del tercer sector.

Resulta evidente que este concepto suele abarcar una amplia y diferenciada gama de cuestiones relativas a los recursos y procesos, vinculados al entramado de relaciones sociales en el cuál se encuentran inscriptos los individuos, y que es considerada sumamente relevante en el desarrollo de acciones, intervenciones y proyectos colectivos.

Según la CEPAL existen “dos dimensiones o ejes principales” en que se pueden englobar las distintas definiciones de capital social: la primera es entendida como la "capacidad específica de movilización de determinados recursos por parte de un grupo" (liderazgo y empowerment); la segunda remite a "la disponibilidad de redes de relaciones sociales" (asociatividad) (CEPAL 2001:3).

En este sentido, entre las definiciones más utilizadas, se considera que el capital social de un grupo social consistiría en la "capacidad efectiva de movilizar productivamente y en beneficio del conjunto, los recursos asociativos que radican en las distintas redes sociales a las que tienen acceso los miembros del grupo" (Atria 2002:5).

Desde ya, existe un amplio abanico de definiciones y especificaciones de este concepto, que hace referencia a una diversidad de cuestiones. En relación con los grupos sociales, las acciones colectivas y, más específicamente, en relación con las políticas y programas de disminución de la pobreza, este concepto tiende a centrarse en la capacidad y potenciación de las acciones cuando están inscriptas dentro de un entramado social más o menos institucionalizado.

En general, en la mayor parte de la literatura sobre el tema, existe consenso en el reconocimiento de dos principales antecedentes en la ela- 
boración de este concepto, tanto las formulaciones teóricas de Bourdieu, como las definiciones desarrolladas por Coleman (Portes 1998, Marsden 2005). Ambas refieren a marcos teóricos y epistemológicos distintos, y las derivaciones tanto de sus tratamientos sistemáticos como la descontextualización de sus definiciones, tienen importantes consecuencias tanto en relación con la conceptualización de la problemática abordada como con el carácter de las prescripciones políticas que de ellas surgen.

Evidentemente las diferencias de estos abordajes son notorias, y se amplían aún más en la medida en que la diversidad de usos y aplicaciones se distancian cada vez más de los marcos teóricos en los que fueron formulados. En definitiva, resulta importante recontextualizar la noción de capital social dentro del sistema teórico en el que se haya inscripto - tanto en el caso de Bourdieu en su teoría de las prácticas que transforman y son especificadas por la estructura social, como en el caso de Coleman, en la función del capital social en la conformación de capital humano- para no caer en una circularidad lógica, -como bien señala Portes-, en donde el capital social sea simultáneamente tanto causa como efecto de lo que se pretende explicar. Esto resulta claro cuando se establece ex post que determinados proyectos, políticas o prácticas fueron exitosas mediante el fortalecimiento, creación o utilización del capital social, aunque no resulta claro si, utilizado de este modo, el capital social es una condición de su ocurrencia o es un efecto de esta intervención. (Portes 1998)

Este tipo de tautología sólo es posible de ser superada mediante la inscripción de esta noción dentro de un sistema teórico más amplio, que le otorgue mayor capacidad heurística. Sin lo cual la noción de capital social se convertiría en un concepto pantalla que tiende a ocultar más de lo que permite explicar.

Si tomamos la definición de Woolcock que establece que "el capital social son las normas y redes que permiten a los individuos actuar colectivamente" (Woolcock 2000:3), la misma se inscribe en la crítica al individualismo metodológico del pensamiento económico neoclásico en referencia al desarrollo económico, pero no nos dice mucho más al respecto que las corrientes clásicas de la sociología (Durkheim, Weber, etc.) en relación a la inscripción y conformación del individuo y su accionar dentro de un entramado de relaciones sociales duraderas.

En este sentido cabe señalar que la definición de capital social desde este punto de vista (compartida por Coleman y Putnam) implica, como lo indica J. Sobel (2002:139), la delimitación de un concepto sociológico, pero desde la perspectiva de la economía. Es decir, que si bien define un concepto "sociológico", tal definición se constituye como parte de una interpretación desde la perspectiva económica. Es en el diálogo entre la sociología y la economía en donde es posible contextualizar esta definición.

En cuanto a la delimitación de este concepto desde la perspectiva de las formulaciones teóricas de Bourdieu, el capital social no puede ser com- 
prendido y analizado por fuera del contexto de que es un tipo particular de capital, que se constituye y conforma en interrelación con las otras especies de capital (económico, cultural, político, simbólico) en términos de la definición Bourdesiana. .

En este sentido, en el sistema teórico de Bourdieu, los distintos tipos de capital nunca se manifiestan (en las prácticas de los individuos y grupos) en estado puro sino bajo distintas combinaciones y proporciones; con lo cual, dentro de este marco teórico, no resulta plausible considerar a cada uno de ellos en forma aislada, porque la combinación e interrelación de estos distintos tipos de capital, así considerados, es lo que configura la dinámica de los diferentes campos y la inserción y principio de dominación de la estructura social.

Entendiéndolo de este modo, si se pretende analizar la incidencia del capital social en los procesos sociales tendientes a la mejora de la calidad de vida de los sectores sociales más postergados, debería considerarse la configuración de este tipo de capital en función de su combinación e interrelación con los distintos tipos de capital en un momento socio-histórico determinado y en el contexto del campo de las políticas sociales.

En definitiva, las dimensiones principales que suelen considerar las definiciones del capital social por parte de los organismos internacionales, implican necesariamente cuestiones que exceden el ámbito específico de su definición sociológica.

Tanto la "capacidad de movilización” como la "disponibilidad de redes" (Sobel 2002:151), son cuestiones que remiten inevitablemente a las características del campo político en donde el capital social se valoriza (es decir, establece sus condiciones de existencia como tal); y, por lo tanto, necesariamente refieren a la configuración y tipo de capital simbólico en juego dentro de un contexto socio-histórico determinado.

Tal cuestión significa que la identidad individual y colectiva, las condiciones en que se establecen la legitimidad de determinadas prácticas y grupos sociales, y, los principios de visión y de división de los mismos respecto a las capacidades y disponibilidades propias y ajenas, son cuestiones determinantes -entre otras- en la conformación y características del capital social.

En este sentido, si consideramos el concepto de capital social en forma aislada de las condiciones sociológicas de su conformación y valoración, perdemos de vista la especificidad de su incidencia.

En definitiva, hablar de capital social inevitablemente implica referirse a un proceso de valorización social de determinadas condiciones que exceden su especificidad y, por lo tanto, deben remitir a las condiciones simbólicas en que se realiza, dentro de una configuración de poder (campo político) y principios de dominación específicos. 
Esto significa que el capital social de un determinado grupo de los sectores sociales más vulnerables, se configura también en función de la capacidad que tiene este mismo grupo de hacer visibles y significativas socialmente sus condiciones de existencia para, de este modo, transformar simbólicamente su deprivación (como un modo de apreciar y percibir sus propias circunstancias), en capacidades y disponibilidades vinculadas al recurso de estar juntos (actuar colectivamente).

Esta transformación simbólica es el resultado, necesariamente, de una apuesta de luchas sociales en la medida en que implica enfrentarse a unos principios de visión y de división (a un habitus en términos de Bourdieu) dominantes que tienden a definir a los sectores sociales pobres en función de la carencia de recursos.

En este sentido, casi todas las definiciones de capital social enunciadas desde la perspectiva norteamericana del rational choice (Coleman 1988, Woolcock 2000) refieren al mismo desde la idea de que se constituye en un recurso valioso para las prácticas del individuo fundamentalmente en el desempeño económico, en la medida en que los diversos mercados también se configuran en términos de redes. En definitiva la idea de que este recurso (social) se constituye en capital (social), está fundada en que presenta condiciones de valoración económica en el mercado.

Ahora bien, si consideramos al capital social como un tipo específico de capital diferente al capital económico, hay que considerar las condiciones específicas en que este recurso (las redes sociales y la capacidad de actuar colectivamente) se convierte en capital, es decir, que se valoriza socialmente permitiendo su acrecentamiento y acumulación.

Evidentemente, la configuración de lo social -en estos términos- no sólo como recurso sino también como capital, implica entender las condiciones de su concreción desde la perspectiva de la economía-política. Es decir, que la transformación de este recurso en capital implica la conformación de condiciones socio-políticas que tornen visible y legítima su valorización. Evidentemente esta perspectiva debe considerar que la conformación de un capital social no puede estar exenta de luchas políticas y de disputa en las relaciones de fuerza de los distintos sectores.

Estas luchas llevadas a cabo por los sectores sociales más postergados son (y deben ser) inevitablemente políticas, porque interpelan a la estructura de poder respecto de los criterios de clasificación relativos a la construcción de poblaciones objeto de políticas sociales.

Cuando desde los ámbitos estatales y los organismos internacionales (supra-estatales) se toma la noción de capital social aislada de las interrelaciones señaladas, implica también una toma de posición política en la medida en que, a través de un uso tecnocrático del concepto, tiende a constituirse en el fundamento de prescripciones de determinadas políticas sociales heterónomas a los grupos sociales más vulnerables sobre procesos 
colectivos autónomos (por lo menos en su definición).

Acaso la conformación de estos grupos sociales como destinatarios de programas e intervenciones de este tipo, sería un modo de capitalización política de su propio capital social, en la medida en que se los constituye en interlocutores reconocidos y legitimados por instancias gubernamentales o internacionales y, por lo tanto, les otorga una "voz" ${ }^{2}$ acotada a las prescripciones surgidas de estos mismo programas.

En definitiva el concepto de capital social, cuando se refiere a su utilización en la prescripción de políticas sociales para disminuir la pobreza, debe implicar necesariamente, una reflexión en torno a cuánto de autónomo o heterónomo respecto a estos grupos sociales tiene el proceso de su configuración y validación.

Desde esta perspectiva, resulta relevante describir y analizar brevemente dos casos de producción de hábitat popular en la ciudad de Buenos Aires llevados a cabo en los últimos años por movimientos sociales y de derechos humanos (por el Movimiento Territorial de Liberación y por la Asociación Madres de Plaza de Mayo) cuyo análisis pone en relieve la dimensión política del capital social como recurso, dentro de procesos sociales relativamente autónomos y en contextos socio-históricos específicos.

\section{De qué hablan los movimientos sociales cuando producen hábitat popular}

En la Ciudad de Buenos Aires, durante la década de 1990, las cooperativas de vivienda, las organizaciones de base y las ONG's, se constituyeron en los principales actores no estatales (junto con los organismos multilaterales) en la producción de vivienda social. A principios de la presente década las circunstancias en las cuales se desarrollaron estos emprendimientos, cambiaron dramáticamente.

Después de la crisis económica y política de 2002, los cambios en los procesos de producción de hábitat popular comenzaron a ser más evidentes en el marco de unas precarizadas instituciones públicas, de un sistema político deslegitimizado y el ascenso y consolidación de nuevos actores sociales (movimiento piquetero, asambleas barriales, movimiento de desocupados, etc.).

Como parte de una estrategia en el proceso de reconstrucción del consenso social y de la legitimidad institucional perdida, el Estado comenzó a abrir canales de diálogo con determinados movimientos sociales que se habían convertido en actores políticos relevantes durante el peor momento de la crisis: las organizaciones piqueteras y el movimiento de derechos humanos. 
Frente al crecimiento de la demanda social de viviendas, como consecuencia del empobrecimiento de importantes sectores sociales de bajos ingresos, y la apertura de las instituciones gubernamentales (tanto a escala nacional como local) a la participación de actores sociales, como una estrategia para reconstruir su legitimidad; algunos movimientos sociales organizados desarrollaron proyectos de vivienda social como un modo de aprovechar ese contexto de mayor apertura del Estado y del reconocimiento de los mismos como actores relevantes en la mediación y ejecución de las políticas sociales.

Algunos actores de estos movimientos sociales (fundamentalmente el movimiento piquetero y el de derechos humanos) con una fuerte presencia pública y un relevante carácter político, se implicaron directamente en la producción de vivienda social a partir de constituirse -en ese contextoen legítimos destinatarios y mediadores de las políticas sociales (con financiamiento público).

Las experiencias de los proyectos llevados a cabo por la organización piquetera Movimiento Territorial de Liberación (MTL) y la Asociación Madres de Plaza de Mayo, se constituyen en ejemplos de esta tendencia ${ }^{3}$.

Una cuestión relevante de estos proyectos es que a escala local (Ciudad de Buenos Aires) -en conjunto- implican una producción de vivienda altamente significativa, que incluso supera el promedio anual de construcción del gobierno local (290 por año promedio ejecutados por el Instituto de la Vivienda de la Ciudad hasta el $2007^{4}$ ).

En el caso del MTL, el Proyecto Monteagudo implicó la producción de 326 unidades de vivienda entre mediados de 2006 y el 2007 y la Asociación Madres de Plaza de Mayo a través del proyecto "Sueños Compartidos" ha desarrollado una intensa actividad de producción de vivienda dentro de la Ciudad de Buenos Aires con más de 1.000 viviendas ya terminadas y alrededor de 500 en ejecución en los Barrios Castañares, Los Piletones y Villa 15. Así mismo ha tenido una no menos relevante actuación en la construcción de viviendas en otros partidos del Área Metropolitana y en el resto del país, alcanzando, desde su fundación en 2006, a incorporar al trabajo formal más de 5000 trabajadores y totalizando la construcción de 4800 viviendas, 6 hospitales, 2 centros de salud y un jardín de infantes. ${ }^{5}$

Estos emprendimientos tienen en común que son realizados por organizaciones sociales que se encuentran enmarcadas en movimientos políticos con una fuerte tradición de demandas en la esfera pública. En los últimos años, a partir de transformaciones en sus medios de acción y en sus orientaciones, van configurando una particular modalidad de intervención en la producción de hábitat urbano popular.

Es importante destacar que ambas experiencias no conforman un conjunto homogéneo de acciones y prácticas. Si bien resulta común el he- 
cho de que ambos casos implican la acción de una organización social y política con fuerte presencia pública y que con anterioridad no habían desarrollado acciones en relación con el hábitat; cada proyecto presenta divergencias, tanto respecto a la fuente de su financiamiento ${ }^{6}$ como a las lógicas de su intervención e inserción política.

Probablemente, el factor común que indica su peculiaridad, se refiera a la modalidad fuertemente política en la cual asumen su accionar social para la producción de condiciones habitacionales. Tal vez esto se deba a que son organizaciones sociales que se fueron constituyendo y consolidando bajo el signo de las demandas y protestas en la esfera pública, y teniendo siempre al Estado como interlocutor y “contendiente” privilegiado ${ }^{7}$. Esta situación les ha permitido construir una tradición de participación política en la opinión pública que, a lo largo de los años, les ha otorgado -en ambos casos- una importante legitimidad social como actor político no partidario.

Entonces, resulta posible afirmar que, en términos de Hirschmann (1982), estas organizaciones (piqueteras y de derechos humanos) han construido socialmente una voice $e^{8}$ simbólicamente relevante para el conjunto, en referencia a las problemáticas sobre las que luchan.

Justamente es a partir de esta fuerte estrategia de tipo voice que se han ido constituyendo en actores sociales activos en la producción de hábitat popular. Por tal motivo, es posible afirmar que la dimensión política de estas actuaciones se convierte en la clave para comprender ciertas condiciones de eficacia en la concreción de estos proyectos de vivienda.

Estos emprendimientos, al estar enmarcados dentro de movimientos sociales con una fuerte organización política (como el Movimiento Piquetero en el primer caso y el Movimiento de Derechos Humanos en el segundo) conforman estrategias con un mayor énfasis en la voice, lo que les otorga una importante capacidad de interlocución, tanto con el Estado como con la opinión pública.

Esta particular situación les brinda la posibilidad de construir mediaciones entre los miembros del movimiento que participan en estos proyectos y el Estado, lo que a su vez funciona como una garantía de control y orden social hacia dentro y hacia fuera de las organizaciones, otorgando previsibilidad a la continuidad y concreción de los emprendimientos.

Es decir que una organización social con una fuerte tradición, presencia y voice pública, otorga a su accionar una relativa garantía de continuidad en la medida en que se constituye en un actor político, socialmente legítimo (más allá de las apreciaciones valorativas que los distintos sectores sociales tengan sobre los mismos).

Así mismo, la realización de estos proyectos -a partir del uso eficaz de la voice- tiene la virtud de generar mayor lealtad entre los miembros del movimiento (no sin conflictividad), debido principalmente a que permite 
construir una expectativa de mejora en relación a la permanencia dentro de la organización.

Desde esta perspectiva no es casual el hecho de que los proyectos señalados superen la pequeña escala y se diferencien de la modalidad de autoconstrucción, en la medida en que su carácter político les permite luchar más eficazmente en la obtención de recursos económicos (créditos, subsidios, etc.), no económicos (servicios profesionales, asesorías gratuitas, etc.) y de financiamiento.

En definitiva, estos actores sociales asumen plenamente su carácter político (en sentido amplio ${ }^{9}$ ) a partir de un uso eficaz de su voice y con ello construyen un enpowerment vinculado a su legitimidad social, a la lealtad de sus miembros y a las capacidades de interlocución con el Estado.

De esa manera, al incorporar la dimensión política en el desarrollo de los emprendimientos, tienden a configurar una alternativa a las modalidades tecnocráticas, tanto en el diseño y localización como en la asignación de las unidades de vivienda a las familias. Tal circunstancia les otorgaría un mayor nivel de sustentabilidad social y simbólica en la consecución de los proyectos.

\section{Capital Político ¿empowerment o valorización del Capital Social?}

Considerando las características señaladas de estas experiencias de hábitat popular y las apreciaciones en relación con el concepto de capital social, resulta posible afirmar que para que el capital social se constituya en un recurso disponible y capaz de ser movilizado dentro de procesos colectivos, resulta necesaria una capitalización simbólica previa ${ }^{10}$.

Tal capitalización simbólica, permite al grupo en cuestión dotarse de las apreciaciones y percepciones tanto de su condición identitaria colectiva (la conformación de un nosotros) como de la valorización del recurso de actuar colectivamente y de su capacidad de desarrollar una voice pública como interlocutor válido y reconocido por el Estado y/o por las instituciones supra-estatales.

Desde esta perspectiva, la constitución de un capital social disponible no sólo como recurso de acción sino también como valor de intercambio en la lucha para la obtención de otro tipo de recursos (económicos, financieros, etc.), implica también la construcción de un capital simbólico de reconocimiento y, con ello, la conformación de un capital político, en la medida en que este reconocimiento implica una apuesta de luchas en la esfera pública frente a los principios de visión y de división dominantes y en la legitimación de la capacidad de interlocución con el Estado y los demás actores sociales. 
En este sentido, el “capital político” se constituiría en un elemento a considerar para la comprensión de los fenómenos colectivos de producción de hábitat popular, en la medida en que la consecución de los mismos implica -como se ha indicado en el punto anterior-, una lucha simbólica por el reconocimiento como actor válido y una lucha política en relación con la apreciación del poder social de estos colectivos en la generación de condiciones de eficacia en la realización de proyectos.

Desde esta perspectiva, dicho concepto englobaría un conjunto -poco analizado hasta ahora- de recursos estratégicos de los movimientos sociales en la generación de condiciones de eficacia en las intervenciones y acciones sociales “a escala” para la producción de hábitat popular y en la reducción de la pobreza y la desigualdad.

Eficacia que se vincula tanto con el poder de movilización de los recursos del capital social como con la sustentabilidad de este accionar en el tiempo. Tal sustentabilidad se relaciona indudablemente con la capacidad y modalidad de los liderazgos hacia dentro de los movimientos o grupos, como así también con la capacidad de representación frente a los demás actores sociales y con las garantías de continuidad organizativa y de su accionar.

En definitiva, es en este sentido que el capital político puede ser entendido en los procesos de producción de hábitat popular, como valorización del capital social cuando, como tal, existe como recurso asociativo y de confianza mutua pero necesita ser reconocido y reconocible en la arena pública para convertirse en un valor que se encuentra disponible como recurso para la acción colectiva.

Entonces, el capital social se constituye en recurso, a través de una estructura de poder hacia dentro del grupo (como representación y liderazgo) y hacia afuera (como reconocimiento y legitimidad social) mediante transformaciones de los principios de visión y apreciación (como resultado de luchas internas y externas), brindando capacidad de ejecución, eficacia en los resultados y sustentabilidad del proceso.

\section{Conclusiones}

Como se ha dicho, la noción de capital social se encuentra ampliamente difundida en Latinoamérica -a escala local, nacional y supra-nacional- como fundamento y recomendación en la aplicación de políticas sociales destinadas a la disminución de la pobreza, entre las que se encuentran los programas y emprendimientos de vivienda social.

La intención del presente artículo es señalar los límites que la disparidad de definiciones y el uso ambiguo de este término impone a su capacidad interpretativa y explicativa, en relación con los complejos procesos sociales en que se inscribe. 
Se ha intentado ejemplificar esta cuestión con dos experiencias relevantes que se encuentran en curso en la ciudad de Buenos Aires. De las mismas se desprende que la capacidad de movilización y disponibilidad del recurso vinculado al capital social, implica una apuesta de luchas en donde lo político juega un rol relevante.

Entendiendo el capital político como la valorización de un recurso ligado a la construcción de una voice pública, a la conformación de un liderazgo interno y a una legitimidad externa frente al Estado y a las demás instituciones y actores sociales.

La posibilidad de que las intervenciones de los movimientos sociales y las organizaciones de base en la producción de hábitat popular actúen a mayor escala y se constituyan en un agente de desarrollo urbano "desde abajo", depende en gran medida del desarrollo de su capacidad operativa, de la consecución de resultados concretos y de su sustentabilidad a largo plazo como actor urbano; cuestiones que irremediablemente refieren al desarrollo y consolidación de un relevante capital político que les permita simbólicamente poner en valor el capital social. 


\section{Notas}

* Este artículo presenta los resultados de una investigación realizada en el Instituto Superior de Urbanismo FADU - UBA entre los años 2006 a 2009. Algunas de las reflexiones que aparecen en el mismo fueron presentadas en el XXVII Congreso ALAS Latinoamérica Interrogada, Buenos Aires, Septiembre 2009.

1 “...estas fuerzas asociativas no son suficientes en sí mismas para producir un impacto eficaz y romper el círculo vicioso de reproducción de la exclusión y la pobreza” (Ocampo 2001:30)

\section{${ }^{2}$ Hirschman (1982:74)}

${ }^{3}$ Para una descripción y análisis más detallado de estas experiencias que fundamentan las interpretaciones que aquí se vierten, ver Scheinsohn y Cabrera (2007 y 2009)

${ }^{4}$ Desde el año 2007 hasta la actualidad la producción de vivienda del IVC fue prácticamente nula y sólo se dedicó a terminar las obras iniciadas con anterioridad.

${ }^{5}$ Ver http://www.suenoscompartidos.org.ar/obras/10-guia-de-obras/

${ }^{6}$ En el caso del Proyecto Monteagudo (MTL) se financió a través del Programa de Autogestión de la Vivienda dependiente del Instituto de la Vivienda (IVC) del Gobierno de la Ciudad de Buenos Aires. Este Programa surge como consecuencia de la promulgación de la Ley 341/ 00 que permite otorgar financiamiento a colectivos organizados, bajo la forma de cooperativas. Estas leyes surgieron luego de una fuerte demanda de diversas ONG's, organizaciones de base y cooperativas dentro de un contexto de mayor permeabilidad del Estado local . En relación con los proyectos de la Asociación Madres de Plaza de Mayo, son financiados a partir del Programa Federal de Construcción de Vivienda del Estado Nacional, que de forma descentralizada otorga los fondos para la ejecución de los proyectos.

${ }^{7}$ Tanto las organizaciones piqueteras como las organizaciones de derechos humanos -especialmente la organización Madres de Plaza de Mayo- tuvieron desde sus inicios un fuerte accionar público que fundamentalmente pretendía otorgar visibilidad social a problemáticas y demandas que el Estado, y mas precisamente el establishment de turno, sistemáticamente pretendían opacar, ocultar y silenciar.

8 "Los consumidores decepcionados tienen otra opción: pueden alzar su voice y participar así en diversas acciones que van desde la queja estrictamente privada hasta la acción pública en aras del interés general”. Hirschman (1982:74)

${ }^{9}$ No sólo como participación en la escena pública sino también en relación con las estructuras de poder.

10 “....aunque efectivamente [las] redes asociativas pueden fortalecer la «integración» de la comunidad, barrio u organización, no bastan para crear suficiente capital social que vincule, a modo de «puente», dicha integración con los sistemas sociopolíticos local, regional y nacional, que son precisamente los ámbitos en los que el capital social de los grupos más favorecidos es usado en estrategias para mantener las condiciones de privilegio, de una generación a otra.”, (Ocampo 2001:31) 


\section{Bibliografía}

Atria, R. (2002), “Capital Social: concepto, dimensiones y estrategias para su desarrollo”, en Capital social y reducción de la pobreza en América Latina y el Caribe, Informe de la CEPAL, Santiago de Chile.

Casey, K. (2005), Defining Political Capital: A Reconsideration of Bourdieu's Interconvertibility Theory, University of Missouri-St Louis, Saint Louis.

Bourdieu, P. (2005), “De la casa del rey a la razón de Estado. Un modelo de la genesis del campo burocrático” en El misterio del ministerio, Gedisa, Barcelona.

Idem (2001), Las estructuras sociales de la economía, Manantial, Buenos Aires.

Coleman, J. (1988), “Social capital in the creation of human capital”, en American Journal of Sociology N 94 pp 95-120. JSTOR, Chicago.

Comisión Económica para América Latina y el Caribe - CEPAL (2001), Capital Social y reducción de la pobreza: en busca de un nuevo paradigma, CEPAL, Santiago de Chile.

Gutiérrez, A. (2005), "Poder y representaciones: elementos para la construcción del campo político en la teoría de Bourdieu”, en Revista Complutense de Educación, Vol. 16 Num. 2, Madrid.

Hirschman, A. (1982) Shifting Involvements. Private Interest and Public Action, Princeton.

Marsden, P. (2005), “The Sociology of James S. Coleman” en Annual Review of Sociology, Vol. 31 pp 1-25, Palo Alto, USA.

Ocampo, J. (2001) “Capital social y agenda de desarrollo”, en Capital Social y reducción de la pobreza: en busca de un nuevo paradigma, CEPAL, Santiago de Chile.

Portes, A. (1998), "Social Capital: Its Origins and Applications in Modern Sociology”, en Annual Review of Sociology, Vol. 3124 pp 1-24, Palo Alto, USA.

Putnam, R. (1995), “Bowling Alone: America’s declining social capital”, en Journal of Democracy $\mathrm{N}^{\circ} 6$ pp. 65-78, JD, Washington DC.

Scheinsohn, M. et al. (2006), “Constructing from within a Social Movement: Self-management of Large Building Enterprises”, en M. Balbo (comp.) Promoting social inclusion in urban areas: policies and practice, Istituto Universitario di Architettura di Venezia. N-Aerus Conference 2005, SIDA/ 
HDM-University of Lund, Venezia.

Scheinsohn, M. y Cabrera, C. (2007), Un particular conjunto de vivienda popular en Buenos Aires. Análisis socio-urbano del "Proyecto Monteagudo”. Ponencia presentada en el XXVI Congreso de la Asociación Latinoamericana de Sociología (ALAS), Agosto de 2007, Guadalajara, México.

Ídem (2007), "Social Movement and Habitat Production in Buenos Aires. When Policies are effective”, 8th N-AERUS Conference, 6th-8th September 2007, London, UK, Web page: http://www.n-aerus.net/web/sat/workshops/ 2007/london_2007_papers.htm

Ídem (2009), "Social movements and the production of housing in Buenos Aires; when policies are effective”, en Environment \& Urbanization: City governance and citizen action II, Vol. 21, Num. 1, abril 2009, pp 109-125.

Sobel, J. (2002), “Can we trust social capital?”, en Journal of Economic Literature Vol. XL. American Economic Association Publications, Sydney.

Wacquant, L. (2005), “Poder simbólico y práctica democrática”, en El misterio del ministerio. Gedisa, Barcelona

Woolcock M. and Narayan D. (2000), “Social Capital: Implications for Development Theory, Research and Policy”, en World Bank Research Observer Vol. 15, Washington.

Recibido: 21.05.2011

Aceptado: 06.07.2011 